\title{
The COVID-19 pandemic and dentistry: the clinical, legal and economic consequences - part 2: consequences of withholding dental care
}

\author{
Paul Coulthard, ${ }_{1}$ Peter Thomson, ${ }^{2}$ Manas Dave, ${ }^{\star 3}$ Francesca P. Coulthard ${ }_{1}^{4}$ Noha Seoudi ${ }^{5}$ and Mike Hilll
}

\section{Key points}

Natural history of oral disease progression if not

managed is described.

Concerns about the medico-legal consequences of reduced access to dental care are discussed.
The economic repercussions of the COVID-19 need for suspension of routine care and the future increased costs of practice are presented.

\begin{abstract}
As routine care was suspended in most countries, one could anticipate progression of undiagnosed and managed oral disease. Patients are usually unaware of the development of oral disease as it is not visible to them and largely asymptomatic, especially in its early stages. The natural progression of conditions such as caries and periodontitis is inevitable without diagnosis and management. The full extent of patient harm because of the suspension of routine dental care can only be estimated when routine oral examinations are fully re-established, and even then, we will probably never know the number of individuals impacted or the extent of disease progression and harm.

In first-world countries, there has always been a back-up emergency treatment system for dental problems. For example, in the UK, the safety net for life-threatening swellings and for acute pain relief is the accident and emergency services. This system remained in place during the COVID-19 pandemic. Courts could be expected to understand the coronavirus context and would take this into account should there be a complaint against the clinician about access to care.

The suspension of routine dental care to save lives will lead to the closure of many dental practices due to substantial financial impact. The return to routine care will be slow, with prioritisation of non-aerosol generating procedures while we look to further understand methods to mitigate transmission risk via infected aerosol and spatter. Inevitably, the cost of personal protective equipment and the lower volume of patients pose a continued threat to businesses.
\end{abstract}

\section{Introduction}

The World Health Organisation declared a public health emergency of international concern on 30 January 2020, following identification of the contagious severe acute respiratory syndrome coronavirus 2 (SARSCoV-2). Following this, the impact on the

Professor of Oral and Maxillofacial Surgery, Dean and Director for Dentistry, Barts and The London School of Medicine and Dentistry, Queen Mary University of London, $\mathrm{UK}^{2}{ }^{2}$ Clinical Professor in Oral \& Maxillofacial Surgery, Associate Dean (Clinical Affairs), Director, Prince Philip Dental Hospital, Faculty of Dentistry, University of Hong Kong, Hong Kong; ${ }^{3}$ NIHR Academic Clinical Fellow in Oral and Maxillofacial Pathology, The University of Manchester, UK; ${ }^{4}$ General Dental Practitioner, Thornhill Dental, Dewsbury, UK; ${ }^{5}$ Senior Clinical Lecturer in Oral Microbiology, Barts and The London School of Medicine and Dentistry, Queen Mary University of London, UK:

${ }^{6}$ Barrister, Parklane Plowden Chambers, UK.

*Correspondence to: Manas Dave

Email address: manas.dave@postgrad.manchester.ac.uk

Refereed Paper.

Accepted 2 November 2020

https://doi.org/10.1038/s41415-020-2406-9 provision of dental care has been profound, with routine care restricted or paused for a period around the world. ${ }^{1}$ Patients could be expected to experience progressive dental disease if denied the dental care needed or if delayed for a substantial period of time. Regular dental check-ups are recognised as an important opportunity for oral cancer screening. Missing this opportunity could be expected to impact not only on quality of life but also on general health and life itself.

Dental professionals have wanted to provide care for their patients, but at the same time, appreciate the possible risk of virus transmission to the dental team and the public. Hence, they are obliged to follow the restrictions posed by public health bodies. In the UK, dental professionals have also been concerned about the medico-legal consequences of withholding care that patients clearly require and, without which, may cause patients to come to clinical harm. There are several questions which have been raised. If the dentist works outside the health service guidance, is there a risk of negligence and legal action? If the guidance produced by a government authority is found to have been inappropriate, could there be a legal challenge against those authorities?

A further concern of dental professionals has been the economic impact. The initial restrictions of routine care in the UK was accompanied by profound stress and anxiety about financial viability. This was lessened for some by the announcement of continuing NHS payments in return for delivery of urgent care or redeployment to support medical care. ${ }^{2}$ For those undertaking private clinical practice independent of the NHS, the anxiety continued.

\section{Natural history of dental disease}

Given that routine care was suspended in most countries, one could anticipate progression of undiagnosed and managed oral disease. 
Patients are usually unaware of development of oral pathosis as it is not visible to them and largely asymptomatic, especially in its early stages. The loss of regular oral examination has been a concern for many patients and for dental professionals.

\section{Caries}

Untreated dental caries in permanent teeth is the most common health condition in the world according to the Global Burden of Disease Study (2017). ${ }^{3}$ Dental caries is the result of demineralisation of tooth tissue by organic acids, causing breakdown of the tooth structure and bacterial ingress. Mutans streptococci and lactobacilli are the main species which metabolise fermentable carbohydrates (such as glucose, sucrose and fructose) to produce organic acids, predominantly lactic acid. ${ }^{4}$ The release of these organic acids reduces the $\mathrm{pH}$ value below the critical threshold, causing demineralisation (chemical dissolution of hydroxyapatite crystals and proteolytic breakdown of tooth structure) through diffusion of constituent minerals out of the tooth structure. ${ }^{5,6}$ This creates an enamel cavity, which further perpetuates the trapping of food debris and harbours bacteria. ${ }^{7}$ When sufficiently advanced through the enamel, bacteria can permeate through the dentinal tubules and subsequently infiltrate the dental pulp. ${ }^{8}$

The histological hallmarks of irreversible pulpitis include the presence of bacteria and their by-products or infiltration of immune cells. ${ }^{9}$ A host immune response is activated in response to the bacterial ingress, and associated inflammatory cytokines and chemokines are produced to remove the source of infection. ${ }^{10,11}$ However, this process can be detrimental in the preservation of tooth vitality. The immune response involves an increase in vascularity and influx of inflammatory cells, which raises intra-pulpal pressure within a mineralised cavity that does not have the ability to deform and accommodate the increased volume. Increased pressure in certain areas within the dental pulp results in collapse of thin walled veins and venules. This causes stasis of blood and localised ischaemia. The increased pressure can also initiate the inflammatory process in periapical tissues. ${ }^{12}$

The dental pulp contains nociceptive myelinated A-delta and A-beta nerve fibres and unmyelinated $\mathrm{C}$ nerve fibres, which form a plexus of nerves (plexus of Raschkow). ${ }^{13}$ The A-delta and A-beta fibres traverse the predentine zone, with their terminal branches entering a short distance into the dentinal tubules. ${ }^{14}$ Myelination facilitates faster conduction of action potentials and a lower threshold for stimulation. Myelinated nerves are responsible for the initial sharp shooting pain of short duration that can be localised by the patient. The stimulation of unmyelinated C fibres causes a dull, aching pain that is diffuse and referred to a general area rather than a specific tooth, making it challenging to localise by the patient. These nerves use less oxygen and can survive a hypoxic environment for longer in comparison to myelinated nerves, hence they continue to function for longer along the progression of pulp necrosis. ${ }^{13}$

The dentinal tubules maintain an outward flow of fluid from the dental pulp, which is affected by surface stimuli. This fluid displacement activates the A-delta and A-beta nerve fibre receptors in the tubules, and triggers sharp and momentary pain. A greater number of tubules, with a greater width and more patency, has been shown to correlate with increased sensitivity. ${ }^{14}$ Treatment with desensitising agents, dentine adhesive sealers and chemical agents which occlude the dentinal tubules such as sodium fluoride are common modalities of management; however, such treatments have been known to have a finite lifespan. Dentine hypersensitivity does not fulfil the threshold of emergency treatment at urgent dental care centres, ${ }^{15}$ but has been shown to significantly impair oral healthrelated quality of life, affecting diet and oral hygiene practices, with the pain extending to affect self-esteem and self-confidence. ${ }^{16}$

In pulpitis, as the intra-pulpal pressure continues to rise, there can be complete compression of blood vessels at the apical foramen, resulting in total necrosis. ${ }^{12}$ Additionally, the release of intra-cellular lysosomal enzymes by neutrophils are also responsible for the destruction of pulpal tissue and resulting tissue necrosis. ${ }^{9}$ Ultimately, there can be a number of irritants other than dental caries, which can result in pulp inflammation and subsequent death such as restoration breakdown, iatrogenic injury, chemical irritation, non-carious tooth tissue loss and trauma. ${ }^{12}$ The delay in the diagnosis and management of dental caries (both clinically and radiographically) ${ }^{17}$ will result in more advanced lesions at first presentation, including those involving the pulp. Lesions with substantial cavitation can adversely affect restorability and increase the requirement for extensive treatment.
The permeation of bacteria from the dental pulp chamber through the apical foramen and lateral canals into the periradicular tissues can initiate a further inflammatory response. Chronic inflammation can cause asymptomatic disease progression and resorb surrounding bone through osteoclast activation. However, acute inflammation can cause pain and the production of an acute apical abscess. ${ }^{18}$ The purulent exudate formed inside the dental pulp can infiltrate the surrounding medullary bone and collect within soft tissues, resulting in a swelling. This can result in several complications; the most severe include the diffuse spread of infection bilaterally into the sublingual and submandibular spaces, causing Ludwig's angina. The resulting pressure can cause challenges in breathing through airway obstruction, which can be fatal. ${ }^{19}$ The collection of pus in certain spaces such as the submasseteric space can cause trismus and increase the risk of aspiration. This can also pose challenges for airway management. The delay in dental assessments for facial swellings can affect patient quality of life and increase the risk of medical complications.

Veins can have internal valves to prevent the backflow of blood; however, these are commonly absent in the veins of the midface as well as facial and ophthalmic veins. ${ }^{20} \mathrm{~A}$ facial swelling around these vessels can lead to the constriction and stagnation of blood flow, predisposing patients to an increased risk of thrombosis, specifically cavernous sinus thrombosis. Sepsis (synonymous with the terms septicaemia, blood poisoning, sepsis syndrome and severe sepsis) is a lifethreatening organ dysfunction caused by a dysregulated host response to infection. ${ }^{21}$ The invading pathogens have survival mechanisms, which confer resistance to the host immune response. This allows the bacteria to evade immune cells, enter the bloodstream and systemically disseminate along with their bacterial endotoxins and exotoxins, causing direct and indirect organ dysfunction. This can lead to substantial patient morbidity and is a cause of mortality. ${ }^{22}$ There are several case reports of dental conditions causing sepsis such as dental abscesses and caries, ${ }^{23}$ and following tooth extractions. ${ }^{24,25}$ The UK Sepsis Trust has produced a 'sepsis six pathway', ${ }^{26}$ which simplifies the key warning signs, patient assessment and management. Assessing for sepsis is one of the major challenges for dental practitioners undertaking telephone triage. ${ }^{27}$ 


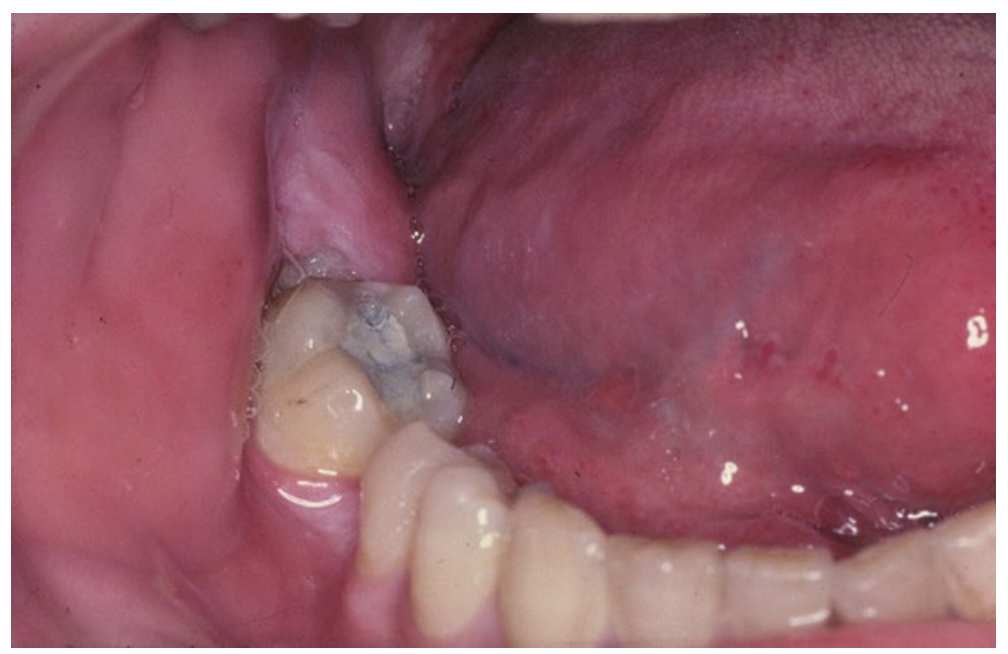

Fig. 1 Pericoronitis of lower right wisdom tooth

There are also generalised risks of analgesia overdose,$^{28}$ heightening dental phobia, ${ }^{29}$ and patients undertaking both interventional oral treatment and repairs of devices themselves with adverse outcomes.

\section{Periodontal disease}

Periodontal abscesses are a common dental emergency and characterised by bacterial invasion of soft tissues around a periodontal pocket, initiating an inflammatory response. This results in a local infiltration of inflammatory cells, destruction of connective tissue and necrosis, and encapsulation of the resulting exudate, leading to a swelling. ${ }^{30}$ Elevated blood lymphocytes and neutrophils as well as cervical lymphadenopathy have been reported in patients, demonstrating a systemic response of this localised infection. ${ }^{31}$ Breach of the oral epithelium through trauma, subgingival impaction of foreign bodies (for example, calculus), orthodontics, iatrogenic conditions and gingival enlargement are some of the factors associated with periodontal abscess formation in patients without preexisting periodontitis. ${ }^{30}$ In patients with periodontitis, a periodontal abscess can be a component of the disease pathophysiology, unsuccessful interventional treatment or the use of antimicrobials. The persistence of a periodontal abscess can lead to greater periodontal attachment loss and adversely affect tooth prognosis. ${ }^{32}$

Necrotising gingivitis is an acute inflammatory processes characterised by areas of necrosis or ulcerations of the interdental papillae. Other symptoms include gingival bleeding, pain, halitosis, fever and lymphadenopathy. If underlying aetiological factors persist or the condition remains untreated, it can spread to the proximal mucosa, to necrotising stomatitis, or cause attachment loss and advance to necrotising periodontitis. ${ }^{33}$ This is an inflammatory process involving the periodontium, where the signs of necrotising gingivitis are present with the addition of rapid bone loss. ${ }^{34}$

\section{Pericoronitis}

Pericoronitis is defined as inflammation of the pericoronal tissues around a partially erupted tooth (Fig. 1). Mandibular third molars are most commonly affected due to accumulation of microbial plaque and food debris beneath the operculum due to challenges in oral hygiene and localised stagnation. This creates a micro-environment favouring the growth of anaerobic pyogenic bacteria causing pain, swelling and trismus. ${ }^{35}$ Bacterial diffusion can cause a collection of pus in soft tissues, leading to further patient morbidity and systemic signs of infection. First-line management is with debridement of the site to remove the source of infection, and adjunct antimicrobials if systemic signs are present. Systemic complications with pyrexia, lymphadenopathy, sepsis and airway management can occur. ${ }^{36}$

There can be no doubt that the natural history of dental disease has progressed in patients during the suspension of routine dental care around the world. In April, in the West Midlands, 10,292 callers were redirected to urgent dental care hubs after calling NHS 111. This translates to one in ten of all NHS 111 calls in the West Midlands being due to a dental problem. ${ }^{37}$ While there has been some attention drawn to individual cases by the media, the full extent can only be estimated when routine oral examination is fully re-established, and even then, we will probably never know the number of individuals impacted or the extent of disease progression and harm experienced because of this suspension of care.

\section{Medico-legal consequences}

\section{Reduced access to treatment}

Patients in pain are of course more likely to face greater hurdles in access to treatment than pre-COVID-19. It is important to remember that, in many countries, there were pre-existing issues with access to treatment in any event. In some countries, it was simply the availability of treatment at all, while in others, it was availability without payment that may or may not have been prohibitive.

In first-world countries, there has always been a back-up emergency treatment system; for example, in the UK, if a patient could not find an NHS dentist and/or could not afford non-NHS dental treatment, then the safety net for life-threatening swelling and for acute pain relief was always accident and emergency (A\&E) attendance. While an A\&E unit may consider the pain caused by (for example) irreversible pulpitis to be something that it could not or should not become involved in, pain relief would invariably be addressed and advice regarding onward treatment provided.

That system remains in place during the pandemic. There may be more robust triage procedures and patients may be more reluctant to attend A\&E units, but the access remains nonetheless. The UK lockdown commenced on 23 March 2020 and, within 17 days, several A\&E units had stressed publicly that they were still open and accessible to those who needed it because attendance dropped by $25 \%{ }^{38}$

In dentistry, there will generally be two sources of the denial of access to treatment the first will be the clinician and the second will be the state in closing the treatment facilities.

\section{Access to treatment denied by a clinician}

Almost all countries have reduced access to elective healthcare across a broad range of disciplines. Elective surgery in hospitals has been postponed to free up bed space for COVID-19 patients. Primary care elective dentistry has been suspended in many countries so as to reduce transmission within the population. That was done in England, not 
by statute, but by guidance. Dental practices were not listed in 'The Health Protection (Coronavirus, Business Closure) (England) Regulations 2020' that came into force on 21 March 2020. On 20 March 2020, NHS England asked practices to 'avoid all aerosol generating procedures (AGPs) wherever possible'. Five days later, on 25 March 2020, NHS England stated: 'All routine, non-urgent dental care including orthodontics should be stopped and deferred until advised otherwise.' ${ }^{2}$ The Care Quality Commission (CQC) adopted the same advice: 1) dentists should not provide routine dental care; 2) dentists should offer telephone triage and advice, giving prescriptions where necessary; and 3) dentists should refer patients who need active emergency treatment to regional urgent care centres where treatment can be provided safely. Subsequently, multiple e-mails were sent by the CQC to dentists to reinforce the aforementioned message.

On 8 April 2020, the General Dental Council (GDC), the UK regulator, advised: 'Refusing to treat a patient when it is not safe to do so is a perfectly proper professional judgement, which we would not look to question. ${ }^{39}$

On that basis, to succeed in a complaint against the clinician (and in this context, that includes an individual hospital), a patient would have to show that it was safe to have been treated and the GDC has made it clear that it would not look to question that judgement. In fact, it would probably have to resile from that to some extent at the extremes. Imagine a case where a dentist simply declared that it was unsafe to treat a patient when thousands of other clinicians were treating in identical circumstances - the GDC would have to review that statement. The reality is that it omitted the word 'readily' or 'lightly' from the end of the sentence.

In terms of litigation as against regulation, the test remains the same: did the clinician act in accordance with a reasonably competent member of the profession? That necessarily imports the factual background that there was a coronavirus pandemic at the time. There is already case law that demonstrates that the courts understand this context. In 'Mulholland v Medway NHS Foundation Trust (2015) EWHC 268 (QB)', Mr Justice Green said that 'context cannot be ignored. The assessment of breach of duty is not an abstract exercise but one formed within a context - which here is that of a busy A\&E where the task of the triaging nurse is to make a quick judgement call as to where next to send the patient.40
If the test does not change, then it is hard to see how the risk of litigation changes unless there is simply a greater level of negligent treatment. Unpalatable though it may seem, there is a 'background level' of negligent treatment and it will be measurable in incidents per day. It is conceivable that clinician fatigue (induced by working longer hours) will increase that background level, but that would not be a concern within a dentistry environment. It is hard to see that there will be an increase in dental litigation as a result of the pandemic. Indeed, the opposite is likely to be true. If the background level of negligence in elective dentistry in the $\mathrm{UK}$ is (to pick a number) $1 / 10,000$ treatment episodes, then a reduction in treatment episodes will necessarily bring about a reduction in negligent treatment incidents and therefore claims exposure.

The existing indemnification arrangements persist throughout the pandemic period for all clinicians, but specifically to address the fears of those who may be re-tasked; for example, the NHS endodontist re-tasked to provide medical support care at a COVID-19 general hospital (Nightingale hospital) - the UK government extended, by way of Section 11 of the Coronavirus Act 2020, an indemnification guarantee.

\section{Access to treatment denied by the state}

If the aggrieved patient can take no action against a clinician who does not treat because the clinician acts in accordance both with his/ her regulatory guidance and in accordance with a reasonably competent member of the profession, can the patient take aim at the state or the national health service of the country involved?

In England and Wales, since 2012, the duty to provide resources for, inter alia, dental services has fallen to clinical commissioning groups 'to such extent as it considers necessary to meet the reasonable requirements' of the population. ${ }^{41}$ The courts have in the past implied the words 'such as can be provided within the resources available' into that section. That is known as the resources defence. The courts do have jurisdiction over resource allocation but it would be exercised 'extremely sparingly'.

Alternatively, in England and Wales, the Human Rights Act 1998 incorporates most of the rights within the European Convention on Human Rights (ECHR). A state or its national health service would necessarily be an emanation of the state, a requirement for a judicial review of a public body. That is the route which the aggrieved patient would have to take, but the textbooks do not abound with cases wherein it can be successfully argued that there is a breach of an ECHR article for failure to provide medical treatment (other than to save life). In the context that emergency treatment remains available to all dental patients in a life-threatening position, ECHR arguments are of academic interest only in dentistry. The National Institute for Health and Care Excellence recently changed its frailty assessments within COVID-19 decision-making when judicial review was threatened on the basis that it discriminated unfairly against patients with pre-existing disabilities. It is hard to see, however, that any such unfair discrimination exists in the decision-making of governments to restrict elective dentistry and AGPs in particular. That is all the more the case in the face of the provision of both 'cold' and 'hot' COVID-19 urgent dental care centres.

Dentists appear to have closely followed the legislation and statutory and state guidance in response to the pandemic. It is hard to envisage a rise in dental negligence litigation; in fact, the less dentistry that is carried out, the less negligence there will be. It is true that some patients will have to wait longer for treatment from an urgent dental care centre than they would have had to from their previous provider. It is also true that some teeth that could have been restored will be extracted as a result of the disinclination to engage in AGPs, but it is unlikely to result in significantly increased action by regulators or lawyers.

\section{Economic consequences}

Balancing saving lives with risk to the economy is a difficult judgement for governments. It is believed that people are likely to instinctively respond to the value of saving lives rather than to calculate harms from economic downturn. The suspension of routine dental care to save lives led to the closure of many dental practices, with substantial financial impact. The initial closing down of routine care in the UK was accompanied by profound stress and anxiety regarding financial viability. This was lessened for some by the announcement of continuing NHS payments in return for delivery of urgent care or redeployment to support medical care. For those undertaking private clinical practice independent of the NHS, there were no NHS payments.

The return to routine care will be slow, with prioritisation of non-AGPs while 
we look to further understand methods to mitigate transmission risk via infected aerosol and spatter. Inevitably, the cost of personal protective equipment and the lower volume of patients pose a continued threat to businesses. The British Dental Association surveyed their memberships and revealed that only $8 \%$ of practices reported that they were confident in maintaining their financial sustainability. The current NHS payment system was planned for reform before the COVID-19 pandemic and so there is now an impetus to accelerate this development. For patients seeking care privately and independent of the NHS, and for dental treatments that are not funded by the NHS, the increased cost will need to be passed to the patient through increased charges. While the UK government's Coronavirus Job Retention Scheme which provides financial support to furloughed workers has been a strong support, some practices will not reopen because of financial collapse.

\section{Summary}

Given that routine care was suspended in most countries, one could anticipate progression of undiagnosed and managed oral disease. Patients are usually unaware of development of oral pathosis as it is not visible to them and largely asymptomatic, especially in its early stages. The natural progression of oral diseases is inevitable without professional diagnosis and management. The full extent of clinical harm because of the suspension of routine dental care can only be estimated when routine oral examination is fully re-established, and even then, we will probably never know the number of individuals impacted or the extent of disease progression and harm. Courts could be expected to understand the coronavirus context and take this into account should there be a complaint against the clinician regarding access to care.

\section{References}

1. Dave M, Seoudi N, Coulthard P. Urgent dental care for patients during the COVID-19 pandemic. Lancet 2020; 395: 1257.

2. Hurley S, Neligan M. Letters, updates and additional guidance for dental teams. 2020. Available online at https://www.england.nhs.uk/coronavirus/publication/ preparedness-letters-for-dental-care/ (accessed July 2020).

3. World Health Organisation. Oral health. 2020. Available at https://www.who.int/news-room/fact-sheets/detail/ oral-health (accessed May 2020).

4. Featherstone J D. Dental caries: a dynamic disease process. Aust Dent J 2008; 53: 286-291.

5. Selwitz R H, Ismail A I, Pitts N B. Dental caries. Lancet 2007; 369: 51-59.

6. Abou Neel E A, Aljabo A, Strange A et al. Demineralization-remineralization dynamics in teeth and bone. Int J Nanomedicine 2016; 11: 4743-4763.

7. Struzycka I. The oral microbiome in dental caries. Pol J Microbiol 2014; 63: 127-135.

8. Pitts N B, Zero D T, Marsh P D et al. Dental caries. Nat Rev Dis Primers 2017; 3: 17030.

9. Gautam S, Galgali SR, Sheethal H S, Priya N S. Pulpal changes associated with advanced periodontal disease: A histopathological study. J Oral Maxillofac Pathol 2017; 21: 58-63.

10. Elsalhy M, Azizieh F, Raghupathy R. Cytokines as diagnostic markers of pulpal inflammation. Int Endod J 2013; 46: 573-580.

11. Rechenberg D K, Galicia J C, Peters O A. Biological Markers for Pulpal Inflammation: A Systematic Review. PLoS One 2016; DOI: 10.1371/journal.pone.0167289.

12. Yu C, Abbott P V. An overview of the dental pulp: its functions and responses to injury. Aust Dent J 2007; 52(1 Suppl): S4-S16

13. Jain N, Gupta A, N M. An insight into neurophysiology of pulpal pain: facts and hypotheses. Korean J Pain 2013; 26: 347-355

14. West N X, Lussi A, Seong J, Hellwig E. Dentin hypersensitivity: pain mechanisms and aetiology of exposed cervical dentin. Clin Oral Investig 2013; 17: 9-19.

15. Hurley S, Neligan M. Preparedness letter for primary dental care. 2020. Available online at https://www.england.nhs. uk/coronavirus/wp-content/uploads/sites/52/2020/03/ issue-3-preparedness-letter-for-primary-dental-care-25march-2020.pdf (accessed March 2020).

16. Bekes K, John M T, Schaller H G, Hirsch C. Oral healthrelated quality of life in patients seeking care for dentin hypersensitivity. J Oral Rehabil 2009; 36: 45-51.

17. Dave M, Coulthard P, Patel N, Seoudi N, Horner K. Letter to the Editor: Use of Dental Radiography in the COVID-19 Pandemic. J Dent Res 2020; DOI: 10.1177/0022034520923323.

18. Nair P N R. Pathogenesis of Apical Periodontitis and the Causes of Endodontic Failures. Crit Rev Oral Biol Med 2004; 15: 348-381.

19. Siqueira J F, Rôças I N. Microbiology and Treatment of Acute Apical Abscesses. Clin Microbiol Rev 2013; 26: 255-273.

20. Zhang J, Stringer M D. Ophthalmic and facial veins are not valveless. Clin Exp Ophthalmol 2010; 38: 502-510.

21. Singer M, Deutschman C S, Seymour C W et al. The Third International Consensus Definitions for Sepsis and Septic Shock (Sepsis-3). JAMA 2016; 315: 801-810.

22. Minasyan H. Sepsis: mechanisms of bacterial injury to the patient. Scand J Trauma Resusc Emerg Med 2019; 27: 19.
23. Carter L, Lowis E. Death from overwhelming odontogenic sepsis: a case report. Br Dent J 2007; 203: 241-242.

24. Lee J J, Hahn L J, Kao T P S L et al. Post-tooth extraction sepsis without locoregional infection - a populationbased study in Taiwan. Oral Dis 2009; 15: 602-607.

25. Moss H, Collier J M, Collier S. An unusual response of denta sepsis to antibiotics: parallels with the Jarisch-Herxheimer reaction. BMJ Case Rep 2012; DOI: 10.1136/bcr.07.2011.4500.

26. The UK Sepsis Trust. ED/AMU Sepsis Screening \& Action Tool. 2018. Available at https://sepsistrust org/wp-content/uploads/2018/06/ED-adult-NICEFinal-1107.pdf (accessed April 2020).

27. Dave M, Barry S, Patel N. Telephone triaging and possible sepsis? Br Dent J 2020; 228: 488.

28. Dave M, Coulthard F, Coulthard P, Patel N. Increased risk of analgesic overdose during the COVID-19 pandemic. Dent Update 2020; 47: 452-453.

29. Armfield J M, Stewart J F, Spencer A J. The vicious cycle of dental fear: exploring the interplay between oral health, service utilization and dental fear. BMC Oral Health 2007; 7: 1

30. Herrera D, Roldán S, Sanz M. The periodontal abscess: a review. J Clin Periodontol 2000; 27: 377-386.

31. Herrera D, Roldán S, González I, Sanz M. The periodontal abscess (I). Clinical and microbiological findings. J Clin Periodontol 2000; 27: 387-394.

32. Herrera $\mathrm{D}$, Retamal-Valdes $\mathrm{B}$, Alonso B, Feres $\mathrm{M}$. Acute periodontal lesions (periodontal abscesses and necrotizing periodontal diseases) and endo-periodontal lesions. J Periodontol 2018; 89(S1): S85-S102.

33. Bermejo-Fenoll A, Sanchez-Perez A. Necrotising periodontal diseases. Med Oral Patol Oral Cir Bucal 2004 9 Suppl: 114-119, 108-114.

34. Papapanou P N, Sanz M, Buduneli N et al. Periodontitis: Consensus report of workgroup 2 of the 2017 World Workshop on the Classification of Periodontal and Peri-Implant Diseases and Conditions. J Clin Periodontol 2018: 45(S20): S162-S170.

35. Wehr C, Cruz G, Young S, Fakhouri W D. An Insight into Acute Pericoronitis and the Need for an Evidence-Based Standard of Care. Dent J (Basel) 2019; 7: 88

36. Renton T, Wilson N H F. Problems with erupting wisdom teeth: signs, symptoms, and management. Br J Gen Pract 2016; DOI: 10.3399/bjgp16X686509.

37. The Dudley Group NHS Foundation Trust. Council of Governors Meeting. 2020. Available at http://www.dgft nhs.uk/wp-content/uploads/2020/06/20-06-26_CoGAgendaPapers-Public FINAL.pdf (accessed July 2020).

38. Thornton J. Covid-19: A\&E visits in England fall by $25 \%$ in week after lockdown. BMJ 2020; 369: m1401.

39. General Dental Council. Responding to COVID-19 latest updates in uncertain times. 2020. Available online at https://www.gdc-uk.org/news-blogs/blog/ detail/blogs/2020/04/08/responding-to-covid-19latest-updates-in-uncertain-times (accessed July 2020)

40. England and Wales High Court (Queen's Bench Division) Decisions. Mulholland v Medway NHS Foundation Trust (2015) EWHC 268 (QB). 2015. Available at https://www. bailii.org/ew/cases/EWHC/QB/2015/268.html (accessed November 2020).

41. NHS England. The National Health Service Commissioning Board and Clinical Commissioning Groups (Responsibilities and Standing Rules) Regulations 2012. 2012. Available at https://www.nhs.uk/choiceintheNHS/Rightsandpledges/ Waitingtimes/Documents/nhs-england-and-ccgregulations.pdf (accessed November 2020). 